\title{
S9. Family education and involvement in mental health
}

FAMILY INTERVENTION AND DIFFERENT PSYCHOPHARMACOTHERAPIES FOR RELAPSE PREVENTION IN SCHIZOPHRENIA

G. Wiedemann, Department of Psychiatry, University of Tuebingen, Tuebingen, Germany

In our study, we investigated the relative effect of behavioral family management in combination with either targeted or maintenance medication on relapse rates and social functioning of the patient as well as coping and burden within the family. 51 patients with 73 relatives were randomly assigned to the targeted or maintenance therapy group. The groups did not differ in sociodemographic or clinical characteristics at intake.

Targeted medication led to a significant reduction of the dose administered at 6,12 , and 18 months. The average daily dose during the first year was $148 \mathrm{mg}$ chlorpromaxine (CPZ)-equivalents (equ.) in the group receiving targeted medication compared to $266 \mathrm{mg} \mathrm{CPZ}$-equ. in the group receiving maintenance medication. Despite such a low dosage, no deterioration in other clinical variables was observed. No differences emerged between the groups concerning psychopathology measured by the BPRS (BPRS total score 22 at 18 months in both groups). The GAS, measuring symptomatology and level of social functioning (GAS 82 vs 84), and social adjustment assessed by the SAS (SAS total score 2.9 in both groups) did not differ significantly between the two groups. Both groups exhibited significant improvements in all variables assessed at 12 and 18 months compared to their pre-treatment status. Relapse rates differed significantly at 18 months, but not at 6 and 12 months.

The findings suggest that psychoeducational family management in combination with standard-dose therapy proved to be effective in preventing relapse in schizophrenia, whereas targeted treatment together with family therapy does not seem to be a viable alternative as a routine outpatient treatment for schizophrenia, at least under these research conditions. However, it may be an alternative for selected patients, e.g. those unwilling to take standard medication for an extended period of time. Possible explanations and clinical implications of these effects are discussed.

\author{
FROM FAMILY TO STAFF - EXPRESSED EMOTIONS IN \\ STAFF \\ L Kuipers, E Moore \\ Institute of Psychiatry, De Crespiony Park. \\ GB-London SE5 8AF, Great Britain.
}

Expressed Emotion (EE) is a well established and reliable predictor of outcome in schizophrenia when clients return to live with families. Staff in long term settings habe to cope with similar behaviour problems and it has now been found that they share similar attitudes, in that they can also be rated as high or low EE (MOORE and KUIPERS 1992).

Such attitudes are related to outcome, reflected in different coping styles and staff interactions with clients. It is also apparent that particular patient behaviours are associated with higher levels of criticism, and that some clients are more likely to be disliked. These findings have implications for clinical management in long term settings where staff attitudes may be particularly important, and these are discussed. 


\section{EMOTIONAL FAMILY FACTORS IN THE COURSE OF SCHIZOPIIRENIC ILLNESS: RESULTS OF AN 8 YEARS FOLLOW-UP}

H.Schulze Mönking, K. Stricker

St.Rochus-Hospital, Postfach 120, D-48283 Telgte, Germany

The expressed emotion index and the emotional familiy atmosphere of 99 chronic schizophrenic outpatients $(72 \mathrm{~m}, 27 \mathrm{f}$, mean age 27 , mean duration of illness 5,5 years) were examined in the framework of the Münster Families-Study, a prospective evaluative intervention study for the influence of therapeutic relatives groups on the course of the illness. Examinations were done at the beginning 1984 and two years later after the relatives had participated in relatives groups for one year.

After 8 years 65 of the families joined the follow-up. The EEindex was a significant predictor for the total time spent in a psychiatric hospital for the 8 year follow-up and even for the follow-up period from 1988/89 - 1992/93, but there was no prediction of the number of rehospitalisations within 8 years. The subscales resignation (MFB), emotional overinvolvement (CFI) and warmth (CFI) correlated significantly with the symptomscore (AMDP) and psychosocial adjustment (GAS-rating) after 8 years. Patients of relatives which had a high level of warmth and lower emotional overinvolvement or no resignation had the best outcome in symptoms and psychosocial adjustment.
TO WHAT EXTENT CAN SCHIZOPHRENIC PATIENTS BEHAVIOUR AND SYMPTOMATOLOGY EXPLAIN RELATIVES. EXPRESSED EMOTION (EE) ?

H Bentsen, TH Notland, et al

University of oslo, Gaustad hospital, 0320 oslo and Blakstad hospital, 1370 Asker, Norway.

objective: To determine 1) whether certain kinds of behaviour are more frequent among schizophrenic patients with high $E E$ relatives than among those with low EE relatives and 2) whether the degree of behaviour deviance is greater among those with high EE relatives.

Methods: 47 patients (schizophrenia, schizophreniform disorder DSM-III-R, 18-39 yrs, admitted in hospital the last week, at least weekly face-to-face contact with relatives) and 72 relatives. Assessment at inclusion and repeated $4 \frac{1}{2}$ and 9 months after discharge from hospital. Instruments: Positive and Negative Syndrome Scale, Clinical Global Impression, Social Performance Schedule, Perceived Family Burden Scale, Camberwell Family Interview, Five Minute Speech Sample.

Results: Data from the inclusion assessment will be presented and discussed. They have not been analyzed yet. The complete abstract with results and conclusion will be sent AEP in February 1994.
PERCEIVED BURDEN AND PHYSICAL COMPLAINTS AMONG FAMILY CAREGIVERS FOR NEUROLOGIC AND PSYCHIATRIC PATIENTS

E Gräsel

Department of Medical Psychology and Psychopathometry, University of Erlangen-Nuremberg, Schwabachanlage 6, D-91054 Erlangen, Germany

The study attemps to analyse the extent to which physical complaints correlate with the degree of burden under which family caregivers stand. The sample covers 114 caregivers ( 98 women, 16 men), aged on average $58(s=13)$, who care for relatives with nursing needs caused by different diseases, whereby dementia patients constitute the largest category $(48 \%)$. The complaints were documented by means of the "Gießener Beschwerdebogen", the burden by means of the "Häusliche Pflege-Skala". The extent of the complaints is expressed by comparison with the normal population by the frequency comparison of percentage ranking (PR) $>50$ versus $P R \leq 50$. An above-average number and range of complaints is evident for the total pressure of complaints $(70 \%$ vs $30 \%, p<.001$ ) as well as for special complaint complexes. The range of the complaints is significantly connected with the degree of subjective burden. The relation in those cases where little burden was registered corresponds to that of normal population ( $48 \%$ vs $52 \%)$, whereas in those of medium burden $(71 \%$ vs $29 \%, \mathrm{p}<$ $.001)$ and in particular those of great burden ( $94 \%$ vs $6 \%, p<.001$ ) a very noticeable increase in the number of physical complaints is apparent. Reducing the burden on family caregivers by supporting them in their work has a positive effect on their health as well as on the probability of subsequent nursing home placement. 